\title{
Research on pulse laser digital power supply control system
}

\author{
Li Xu \\ College of Electronic Information Engineering, Wuhan Polytechnic, Wuhan 430074,Chian
}

Keywords: pulse laser; digital power control; energy stability; welding application.

\begin{abstract}
DSP full digital control in pulse laser welding power source is composed of Boost and Buck two topological structure. Through the ADC of DSP can test the laser energy, and charged the current of laser xenon lamp in real-time. Through the software PI algorithm to adjust the duty cycle of the IGBT in real time, the purpose is output laser energy stably.
\end{abstract}

\section{Introduction}

As a special light source, laser has the characteristics of high brightness 、 high direction 、 high coherence and high monochrome etc. and has been playing a more and more important role in actual production, laser type can be divided into: $\mathrm{CO}_{2}$ laser、 Nd: YAG pulse laser、 fiber laser etc. Pulse laser has many characteristics, such as high power, high photoelectric conversion efficiency, small thermal effect, good mechanical performance, high degree of automation, suitable for continuous and pulsed light etc. And it is widely used in practical production at present ${ }^{[1,2]}$.

In the process of actual production, the stability of the laser energy and the ability to resist the actual temperature difference are highly demanded, but Nd: YAG pulse laser also has certain limitation, that is to say the pulse energy stability is difficult to reach $3 \%$, which limits its application in some fine processing field, such as the application of precision cutting, precision welding etc ${ }^{[3]}$.

Laser welding power source is the key part of a laser welding, when the optical path is at certain circumstances, the effect of laser welding, laser energy stability and laser welding power source have direct relationship. The traditional welding laser power supply mostly adopts the power chip such as TL494 control, but the reality is that multi- block power chip are often involved in the control, which increases the complexity of the circuit. The main disadvantages are as follow : (1) in order to make sure the laser power output stability, energy feedback and current feedback mode, multi -block power supply chip and external component should be matched, but it is difficult to make the area of circuit miniaturized. (2) use of too many discrete devices, production, commissioning, cost, and device parameters drift for a long time will bring negative effects. (3) adjusting discharge characteristics can only by changing the circuit according to the different xenon lamp load ${ }^{[4-5]}$.

This paper adopts the digital control method to research pulse laser welding power source, the whole system only use a DSP core chip control, and has achieved highly integrated compact, besides, debugging process, laser power stability, cost control has a lot of advantages.

\section{Digital power supply control system}

\subsection{The overall system design principle}

The Boost energy storage capacitor charging part of the pulse laser welding power supply and part of the Buck capacitor discharging xenon lamp adopt the DSP2812 software PI digital control mode. The first part: through the DSP with ADC to sample the size of storage capacitor charging current, and then through PI operation to adjust the IGBT duty cycle to realize the goal that a constant flow of energy charges the storage capacitor. The users can edit the required parameters of welding waveform, pulse width, frequency, laser power rate according to the laser touch screen. Since the system uses a 2812 DSP chip, the host computer touch screen provides a lot of digital sampling information, such as the current average discharge current, peak discharge current, the 
laser energy size, input voltage magnitude, energy storage capacitor voltage size and laser output waveform in real-time, all of which greatly facilitate the user.

Full digital control pulse laser power supply mainly uses a DSP software of PI mode to realize the control, system design principle is shown in Figure 1.The DSP software controls the IGBT battery charge and discharge part in the the main power circuit, and then start the feedback process on voltage, capacitance, energy detection, and also feedback protection of the circulating cooling system (liquid level, flow etc.) of a variety of hardware.

In order to improve the running speed, DSP expanded SRAM.AD, which mainly adopts input voltage, high voltage capacitors, capacitor charging current, xenon lamp discharge current, the laser energy size etc. Inputting IO signal liquid level, flow, water quality, temperature alarm, IGBT protection, hardware protection, waveform calls, pedal signal etc. Event manager A and event manager B are respectively used to be PWM output signal. Outputting IO signal status indication, simmer trigger, slow power supply, optical gate control etc. SCI serial communicative part is used for communication with the host computer 485.

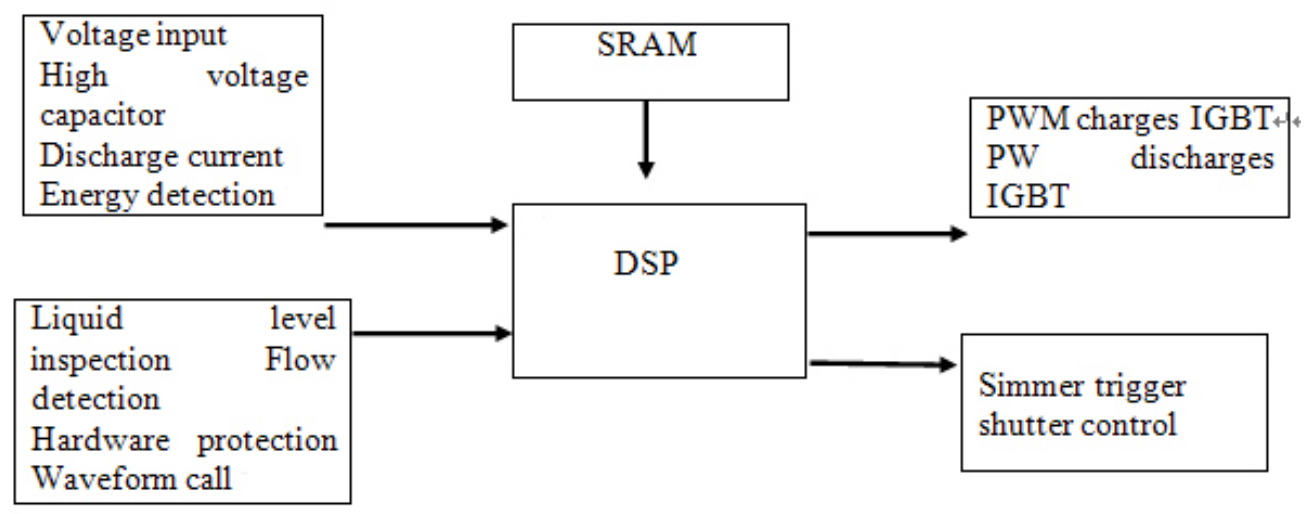

Figure1 The overall system design principle

\subsection{System software core algorithm}

PI core algorithm of the digital control welding pulse power supply software is shown in Figure 2.The well-known PI algorithm is uo $=\mathrm{Kp}^{*}[\mathrm{e}(\mathrm{k})-\mathrm{e}(\mathrm{k}-1)]+\mathrm{Ki}{ }^{*} \mathrm{e}(\mathrm{k})$, uo is the results of PI, Kp and $\mathrm{Ki}$ is proportional and integral terms, e (k) for this error, e (k-1) as the last error.DSP samples the voltage and current and laser energy size, through the calculation results of the PI algorithm above to adjust the duty cycle of IGBT size, which finally can help control the load.

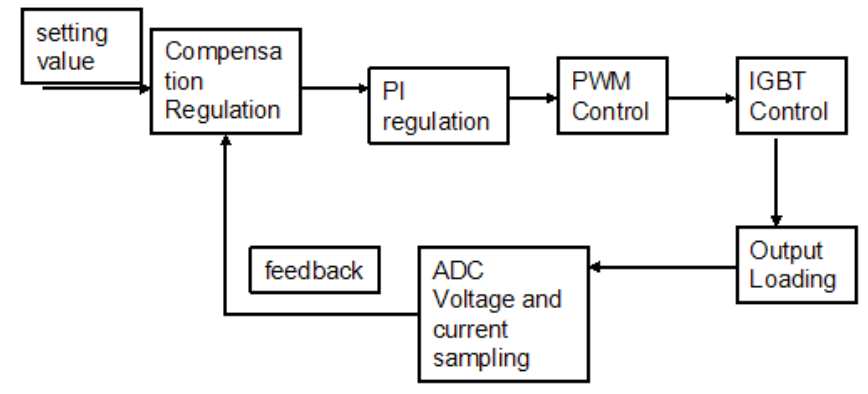

Figure2 The power of software PI core algorithm

\subsection{The overall system flow chart}

Digital control laser pulse power supply software flow chart is shown in Figure 3.First, through the event manager to start the AD sampling in real time, after AD sampling follows interruption, read each passage value, and then call preset PI algorithm to calculate the needed size of duty ratio. Finally, calculate the interrupt flag of completing the $\mathrm{AD}$ and wait for the next $\mathrm{AD}$ interrupt. 


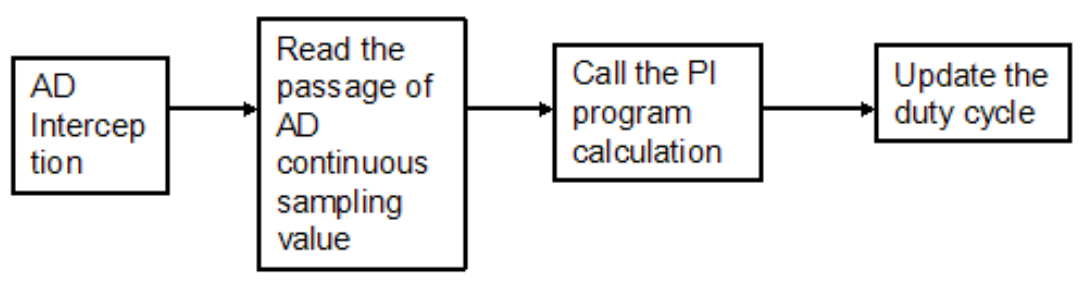

\subsection{Laser output stability}

Figure3 The software flow chart of the power

Using the laser energy meter to start a stability test on laser output energy which average output power is $150 \mathrm{~W}$.To test for 100 times respectively at lower power, medium power and higher power, observing the stability of laser energy. The test results are shown in table 1.

Table 1 the test of stability

\begin{tabular}{|l|l|l|l|l|l|}
\hline Number & $\begin{array}{l}\text { laser peak } \\
\text { power/W }\end{array}$ & $\begin{array}{l}\text { pulse } \\
\text { width/m } \\
\text { s }\end{array}$ & $\begin{array}{l}\text { frequen } \\
\text { cy/Hz }\end{array}$ & $\begin{array}{l}\text { average } \\
\text { power/J }\end{array}$ & instability/\% \\
\hline 1 & 1000 & 5 & 1 & 5.1 & 2.43 \\
\hline 2 & 2000 & 5 & 1 & 11.2 & 1.75 \\
\hline 3 & 3000 & 5 & 1 & 17.28 & 2.59 \\
\hline
\end{tabular}

It was found from the laser energy stability test that no matter under low power (number 1), middle power (number 2) or in high power (number 3), the energy of the laser are stable within 3\%. In middle power laser power, the power is most stable that can reach $1.75 \%$. While under low power and high power, the stability is relatively low, it is possible that laser power supply was limited by its device sensitivity.

\section{Conclusion}

(1)、The whole control system complete all control functions of the main power circuit only by using one DSP core chip, which finally reduce the cost of the components, greatly improve the stability of power supply, increase production debugging, and bring convenient for the use and maintenance of the customer.

(2) 、 Laser output stability of digital control pulse laser power can be controlled within 3\%, and has broad popularization significance for precision laser welding applications.

\section{Reference}

[1]TZENG Y F.Process characterization of pulsed Nd:YAG laser seam welding[J].Advanced Manufacturing Technology ,2000,16(1):10-18.

[2]S.Dadras,M.J.Torkamany,J.Sabbghzadeh.Spectroscopic characterization of low-nickel copper welding with pulsed Nd:YAG laser[J].Opt.lasers Ens.,2008,46(10):769-776.

[3]Rui sheng Huang,Liming Li u,Fan Zhang.Influences of laser in low power YAG laser MAG hybrid welding process[J].Chin.Opt.Lett.,2008,6(1):47-50.

[4]ZHANG Shu-hong,IGBT module application in laser power and improving the performance of laser power[J].Applied laser,2003(2):35-36.

[5]Li Hexuan.The development trend of power electronics technology[J].New Process,2005(6):10-12. 